\title{
Digital Manufacturing: What Are We Able to Print?
}

\author{
A. Muguruza Blanco and J. Minguella-Canela \\ CIM-UPC, Universitat Politècnica de Catalunya-Barcelona TECH, Barcelona 08028, Spain
}

\begin{abstract}
In a rational exercise, in the present paper it is extrapolated how the development of ICTs (information and communication technologies) and the incipient technological development of additive manufacturing has the potential to change our society. In the following, it is analyzing the evolution of man over physical matter and how this has shaped our society. The main milestones or key stages in history that have marked a transcendental change in the human-machine-environment relationship have been identified and consequently have led us to ask ourselves: What is next, how far are we, and what are we capable of printing? In an attempt to identify the current state of the art, highlighting the possibilities those additive technologies can offer.
\end{abstract}

Key words: History, economics, philosophy, digital manufacturing, additive manufacturing, printing electronics.

\section{Introduction}

We are in a time of great changes, there are those who maintain, that in reality we are living the end of an era, which will give way to another that will reinterpret our civilization at all levels. Concepts such as "post-industrial era or society, information, knowledge" or "digital society" are some of the terms that take on more prominence every day and that have been coined in the attempt to identify and understand the scope of the changes introduced by the development of ICTs (information and communication technologies), but while the debate continues in the theoretical field, reality runs ahead and only time will tell the names and terms that we will use to define what we are starting to live in.

Machines, as formal evolutions of tools, have been with us since the beginning of cultural development. First as extensions of our limbs and then; as artefacts independent of the manipulation of man. Even though machines have always accompanied man, they have not related in the same way with him over time.

From the divisions made by the author Alvin Toffler [1] in his book The third wave, where four milestones

Corresponding author: Asier Muguruza Blanco, Ph.D. candidate, industrial engineer, research field: hybrid additive manufacturing technologies for the obtaining of multi-material parts. or key stages in history are established that have marked a transcendental change in the man-machine relationship, in the following analyzing the sequential evolution of man over physical matter and how this has shaped our society. In a rational exercise, it is extrapolated how the development of the ICTs and the incipient technological development of additive manufacturing have the potential to change our society, which leads us to ask ourselves: How far are we and what are we capable of printing (reproduce)?

\section{Milestones of the Human over the Physical Matter and Their Consequences in Our Society}

So attending to these milestones (see Fig. 1), in the first stage the human was related in a simple and direct way with their tools, the relationship "man-tool" was based on experience, through trial and error, and slow accumulation of knowledge to optimize the use of human energy in the performance of a function. The cooking of food in this sense allowed the assimilation of nutrients in an accelerated way, which ultimately boosted the physical development of man, and with the invention of fire, the useful prolongation of the hours of the day.

Subsequently, with the settlement of humans through agriculture, the possibilities for innovation 


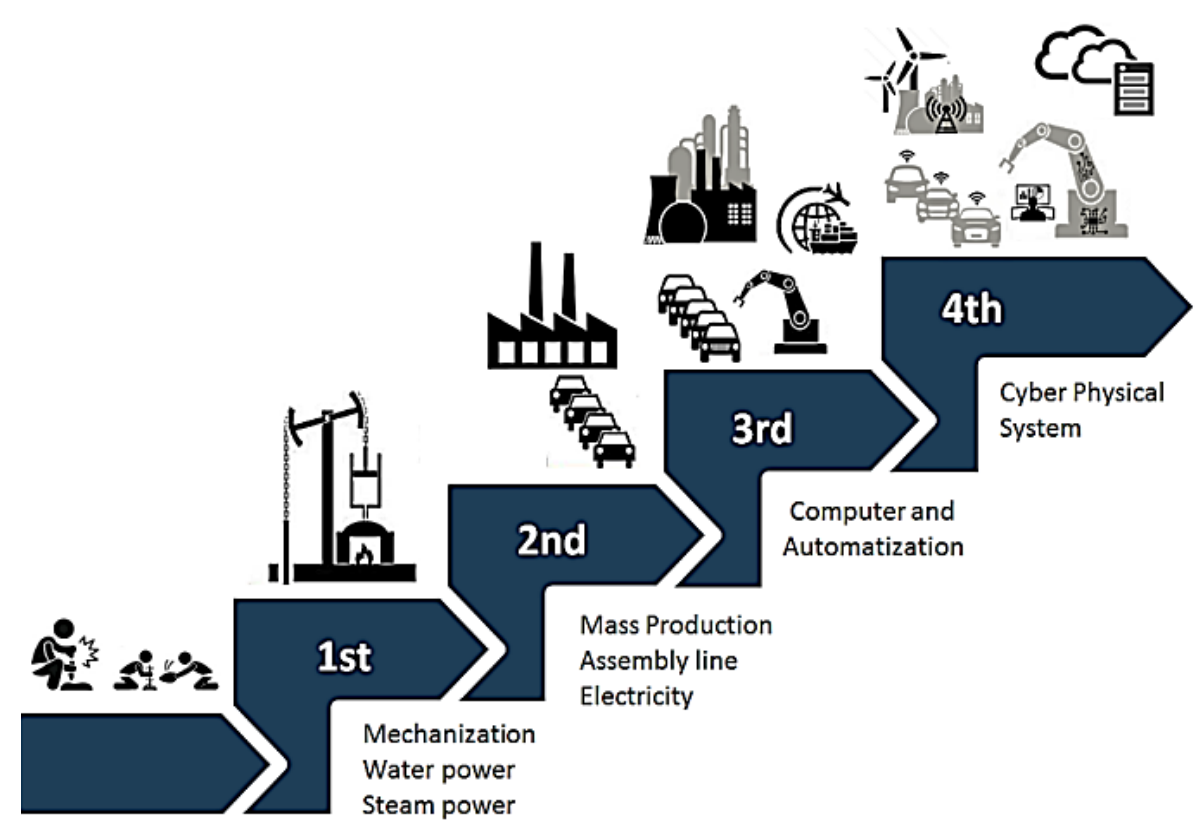

Fig. 1 Industrial revolutions.

multiplied and, in turn, we entered the second historical stage. The establishments arose, with it the villages, towns and finally the incipient cities. Historical records attest to the number of innovations that arose in their path in terms of mechanisms, technics, science and technology. With the writing, the mathematical progress, the arithmetic, the algebra, the trigonometry and the beginning of the documentation of the knowledge, its reproduction evolved the technique to give way to the scientific method (grounded in the systematic observation, the measurement, the experimentation, the formulation, analysis and modification of hypotheses) that is; a philosophy based on irrefutable mathematical facts. On the other hand, the tools evolved into machines (gadgets capable of transforming energy to perform a job). And with the Renaissance, the illustration, the time of the lights, the industry and the commerce, it began to cook the broth of culture of the third stage of the history, the industrial revolution.

It was called revolution because, in effect, it transformed in a radical way the mode in which we lived, we consumed, and we perceived existence. Supported in modernism, and with the idea of "progress", the industrial revolution exploited the full potential of capitalism of production, and also, the amount of technical devices necessary for mass production of consumer goods and with the democratization of products, the artisan disappears, the concept of functional worker appears and the systemic division of tasks in a sequence of processes in order to maximize their efficiency, we enter into Taylorism and consequently into Fordism. That is to say, at that time, as the history tells, human beings became repeaters of defined movements, inserted in the assembly line. In that moment, the relationship between man and machine was transformed, from being a direct relationship and supported by trial and error, to be a relationship mediated by training, and supported by repetition.

At the time when the industrial revolution began to mass produce objects, not only was the technique democratized but, in turn, the form was democratized as well, since every object, apart from its utilitarian function, has a communicative function, supported by that form that is, its surface. At the beginning of the industrial revolution, geometries had little or no importance at the time of their manufacture. The 
objects were "ugly", devoid of forms that evoked positive emotions in individuals (at that time the most important quality of the objects of mass consumption was its function and cost without having anything to do with beauty). The increase in competitiveness and the need to differentiate from the competitors to the more Darwinian style, led to the democratization of beauty as some call it, setting a historical precedent that forced, to rebuild the products of mass consumption thinking of the way in which the user would interact with them, and how said user would respond to the stimuli that said product would produce.

Terms like ergonomics appeared (focused on both products and processes), the human-machine-environment relationship becomes relevant and, with chain production, the concept of an interchangeable element that triggered standardization. But, while the design advanced in the formal and functional configurations of the objects of mass consumption, the technology also advanced at its own pace, creating new materials, ways of generating energy and new production technologies. With the invention of electronic gadgets like the transistor (which revolutionized the field of electronics), a lot of new tools were developed in order to feed the design and the manufacture of the objects, improving the man-machine relationship, understanding the machine now as an object not as a thing used in the industry. In fact, the transistor, as an apparatus developed in the 50s, was the seed of what can be considered the fourth stage in the history of mankind.

With the transistor, the electronics proceeded to miniaturize and the flow of information that these artefacts now electronic devices could handle was increased. With the evolution of chips (thousands of transistors in a silicon plate), microchips, microprocessors, incipient software and computer science, a new form of human-machine interaction arose, the interaction based on the reading of states, and supported by the historical baggage of previously used products.
From this point, our capacities are accelerated rapidly. The miniaturization of electronics increased the computational power of electronic devices. Things that were unthinkable to date, devices to measure the size of a room, with a computing power that allowed sending man to the moon became available, forming part of homes. This miniaturization and increase in the computational power of the devices gave them characteristics that in the past they did not have, as predictive controls, indicator displays, a certain level of autonomy in front of the man who manipulates them, and the ability to follow orders that are more intuitive and close to human thought. In this case, the human-machine relationship was given thanks to the amount of knowledge stored in the subconscious of individuals, similar products in the past. We refer to artefacts that emit a large amount of information so that the user makes the best decisions regarding its use.

Following this chronology, it becomes increasingly evident that man has a tendency to humanize the world around him. When a human is related to another human, even if they do not speak the same languages, they can communicate with rudimentary symbols and signs, thanks to the fact that both share the same biological nature, and are linked to the same genetic legacy that makes them very close to each other. On the other hand, to understand the world, man uses his senses, his reasoning and his ability to deduce behavior patterns, using himself as a standard element, that is, man anthropomorphizes the world to understand it, because that is how he understands himself and looks for similarities on the outside that allow him to understand the environment from his own sensations. If man tends to anthropomorphize the world, it was expected to do so with objects, and endow them with feelings, give them a "humanoid" form and, above all, try to interact with them as if they were, in fact, quasi-human (the cars, for example, are designed according to their functions but, do the nose, the frontal not have a certain similarity to the face of an animal and does the GPS not speak to us?). 
And this panacea is far from being achieved because, to date, it has been impossible to equip the machines with the cultural sediment shared by all human beings and some animals, because they are biological entities with congruent functional characteristics. We want objects that clearly show how to use them through their interface, that do not need instructions to be used, or training, and that, as far as possible, it is not necessary to use additional knowledge for use, except for the baggage acquired through the time and the manipulation of similar objects (we are looking for a closer, more intimate man-machine relationship).

Miniaturization and advances in electronic and computational sciences increased even more capabilities to modern artefacts, offering us the possibility of even communicating with them, letting them work practically without human interaction, predicting possible user decisions, receiving orders in a "natural way", interpreting human intentions and reacting in a preventive manner in case of an accidental error... that is, we went from a human-machine interaction in which the cultural baggage of similar objects was needed, to one in which the machine itself interprets the human, and removes it partially from the field of decisions. Evidently we approach science fiction but we are surrounded by examples that support this trend, in this sense some already dare to glimpse a future where the machines integrated in our organism will give way to a turning point in humanity; "The singularity" point in history where the separation between man and machine becomes diffuse, and where their relationship becomes inseparable.

Returning to the historical development of this relationship and leaving behind this paragraph, from a productive point of view, Fordism evolved towards Toyotism and oriental philosophies focused on combined work. But, in a more flexible and adjusted way, with more optimized speculative strategies, evolved from a local conception of the world, to a global one, and whose finite character propitiated the entrance of ecology in the development of industrial activities, urging the development of policies respectful with the environment. Consequently, new concepts appear such as; traceability, versatility, recycling, life cycle (term associated with the use of products from the beginning until they overcome their technological obsolescence).

Following this previous evolutionary line, in the sequence of milestones we have left the development of ICTs aside, not because they are less important, but because of their transcendence. Under this concept, electronic and computational sciences are combined with telecommunications. The transmission and reception of signals, information in general and more recently wirelessly, has allowed the generation of a new catalyst in the process of evolving human capabilities, the internet. As a decentralized set of interconnected communication networks of global reach, the Internet is established as a new milestone, allowing that the machine-machine relationship delegate the human being only for certain important decisions. In this aspect recently the term internet of things has arisen that, as a consequence of the anthropomorphizing desire of the human being and of the need of companies to differentiate their products from its competitors, they seek through this concept to provide everyday objects with a connection to the digital world, increasing interaction with the user.

What is currently sought is to avoid the need for training of the man against the artefact, and not only seeks to make the products cheap, but they are beautiful, suitable for use, replaceable, respectful with the environment and recently sought a better interaction with the human, that the artefacts communicate with us at a new level. Although initially, this relationship was superficial through the senses (taste, touch, sight...) now we want this interaction to be more direct, simple and above all more intimate, and with the current existing means to equip the artefacts with this capacity through their link with the digital world.

Additive manufacturing in this sense brings offshoring, and by overcoming the geometric 
constraints imposed by traditional techniques, opens up the possibility of exploiting the individual identity of consumers (personalization) and with multiple materials combined, which after fulfilling a structural function it can offer other functions as the ability to perceive, process, store and transmit information. The achievement of these objectives would imply an unprecedented qualitative leap in human capabilities over the mater.

\section{3D Printing: Rapid Prototyping, Additive Manufacturing}

Year by year since the patent which describes the SLA (stereolithography) was registered in the early 1980s [2], the technological evolution of the AM (additive manufacturing) technics has been nothing less than extraordinary. Starting as a promising set of uncommercialized technologies, during the past of 30 years the global market of the AM technology has experienced growth of two digits. It is expected to exceed $\$ 21$ billion by 2020 [3, 4].

The potential and the possibilities on the other hand, of the physical reproduction of three-dimensional elements from a digital file by the selective addition of materials, have captured the attention of many, making AM a constant research source whose applications grow in variety and importance.

Initially, the elements built by these sets of additive technologies have been fulfilling the function of prototypes in the industry, where designers, engineers, manufacturers have optimized their designs through these tools before taking them into the production. However, nowadays the AM technologies have been earning an important role in many sectors such as: in the automotive, aeronautical or aerospace, a field where parts with topologically optimized complex geometries are common or in the repair of worn elements by the use of hybrid systems. Often in the industries, on the other hand, the AM technologies have been becoming also a complementary process to the well-known traditional manufacturing processes (e.g., vacuum and silicone casting molds) [5]. More recently, the use of 3D printing can be found in the personalized pharmaceutical drugs [6], the construction of electronic devices and sensors [7-10], etc.

\section{Society/Religion/Politics}

Following the established line of thought, additive manufacturing allows the reproduction from a digital file of customizable 3D objects, with a certain geometric limitlessness and with a relatively low human intervention. They propose a total disconnection between the design of the object and its manufacture, although strangely it reduces the distance between the conceivable and the realizable. From a broader point of view, the development of these techniques has the potential of, from the virtual storage of the pieces, the relocation of the productive system. We speak of the rebalancing of the industrial fabric at global level, since it allows reducing the salary advantages of some emerging and underdeveloped countries and even by allowing the location of the productive centers anywhere in the world, has the potential to be inside our homes or at least in the immediate vicinity of the consumer and thereby opens the possibility of cancelling transport costs, transferring the costs associated with manufacturing, storage, energy and the waste to the user or to the final consumer (a kind of interconnected global village, where the prosumers meet their needs), let us say a more radical version of the IKEA concept where the assembly of its products runs on behalf of the consumer, thus allowing lower costs and being more competitive. In this way, it breaks economies of scale, reducing and in some cases, completely eliminating speculative strategies against possible scenarios by companies, allowing the ultra-postponement in the supply chain, that is, the maximum delay of all kinds of investment and productive process at the time of demand.

On the other hand, the union in network of these productive centers supposes an unprecedented advance 
in terms of cooperation, multiplying the means of manufacturing available (imagine an extreme case, an imminent catastrophe, the need to build a dam before an imminent hurricane, flood or an oil spill on the coast capable of destroying a local marine ecosystem for example. Under the principle of DIY (do it yourself) by government decree the cooperation of a community equipped with domestic printers, working in a network, would allow in front of an emergency event, generating the constructive and assemblable elements of the same in a matter of hours from a digital file and since the intervention of the human being in this process is at least scarce, the variability induced by it in the final product is reduced to the minimum focusing the problem only on the materials and their storage).

Thus, the reproduction of physical elements from a digital archive represents a new twist in the capacity of man in his desire to humanize the world, the utopian image of machines manufacturing machines without human intervention belonging to today's science fiction, it does not seem very far if we analyze the chronology of human achievements, a fact that would undoubtedly mean another turning point in the history of humanity or as M. Kaku [11] points out in his book The Physics of the Future in which he predicts the appearance before the turn of the century of what he calls; "The replicator", a domestic device based on nanotechnology capable of reproducing anything (being the term "thing" sufficiently ambiguous to encompass any type of consumable from objects and food to even organs) from the assembly of particles and molecules of the base elements that would constitute the voxels (pixels volumetric) of the thing to be reproduced, unattended and with the efficiency of the machines, that is, in terms of reliability, precision and speed, would be the greatest achievement of engineering and technology, the final culmination of all our struggles ever since we wielded the first tool during prehistory.

While this machine belongs to today's science fiction, in the face of the incipient development of quantum computing through which it would allow dealing with files of enormous dimensions. There are already those, who philosophically considers what could be the consequences of their existence, since, in this sense, there are those who affirm that it could alter the foundations of society itself. All philosophies in this aspect and all social systems are ultimately based on scarcity and poverty. Throughout the history of mankind, this has been the dominant issue that has preoccupied society, shaping our culture, our philosophy, and our religion. In some religions, prosperity is considered as a divine reward and poverty as punishment. In Buddhism, on the contrary, it is based on the universal nature of suffering and on the way to face it. The distribution of wealth also defines society itself. Feudalism is based on preserving the wealth of a small number of aristocrats against the poverty of the peasants. Capitalism is based on the idea that productive people get the reward for their effort in a world where there is ownership of resources. But, if lazy and unproductive individuals could get what they want at the push of a button, capitalism would stop working. An apparatus capable of replicating things and under the hypothesis that we all would have the access to press that button, in this sense wrecks all plans and structures, transforming from top to bottom economies of scale and establishing human relations. The differences between those who have a lot and those who have nothing can disappear under our current interpretation and with it, the notions of social position and political power. In the case of socialism whose mantra we could say that it states: "Give to each according to his capacity; to each according to his contribution", or that of communism: "Give to each according to his capacity; to each according to his need", the existence of the replicating device would allow converting said mantras to: "To each according to his wishes".

Thus, in this hypothetical future where thanks to robots and this wonder and to our eyes, magical reproductive equipment, able to solve the problem of 
scarcity, inequalities and with it struggles, confrontations and conflicts, offering us a society so rich able to live surrounded of a sufficient material well-being to satisfy our needs even if we did not work according to current standards, it is difficult not to ask ourselves, in what we will become as a civilization. In this aspect there are those who raise two extreme situations; in the first scenario, civilization could evolve towards a lazy, capricious and degenerate society. As the second possibility, they pose a scenario where there could be a revolution in the entrepreneurial spirit where, free from the fear of ruining themselves and falling into bankruptcy or poverty, individuals could unleash their initiatives sublimating human talent. Probably both would take place in a less extreme reality; a part of the population would constitute an immovable class of people who would simply refuse to work while others released from the coercion that exerts poverty would pursue creative achievements both scientific and artistic. There is, on the other hand, another hypothetical scenario based on the principle of the caveman, where anthropologists affirm that the character of personas has not changed much in the fundamentals in the last 100,000 years. Therefore, as in those primitive societies where goods and hardships were shared equally, the productive member had rights while the lazy and greedy were marginalized from the tribe. In a world where the human has the replicator, the extrapolation of this model or scheme becomes a possibility where social pressure makes a regulatory function and where being part of the tribe is the reward that justifies a certain effort, which in principle would violate the freedom of the individual.

Fortunately, as the progress seems to be slow there is still a long way to go for the appearance of this hypothetical replication equipment. Fortunately for society, it will have time to debate the implications of this technology and adapt to a new reality in order to maintain its status quo or maybe a better reality.

Meanwhile today we settle for the possibility of reproducing objects from the combination and in different grades of related materials, and thus playing with some properties, although very basic compared with the possibilities that these technologies offer.

\section{Reality...}

First of all, we have to say that, the previous step or what precedes all reproduction is the definition, which comes in turn preceded by knowledge. In this aspect since, as the human interprets the environment through their senses (sight, smell, hearing, taste and touch) and after a wealth of shared and recorded experiences, he has acquired knowledge that has led him to define with more or less accuracy what he perceives, and currently with maximizing tools of said initial sense (a microscope or a telescope, for example, supposes the extension of our eyes allowing us to magnify our sense of sight) such perception has been exponentially increased and consequently the definition of our environment is closer to the reality than it was with our predecessors and therefore will be less than what their future successors can achieve, and with the development of ICT's, the registration and transmission of such perceptions they have been expanded like never before.

We are thus immersed in a sequence of events that have conditioned the current state of the art. In the field that occupies us for being very multidisciplinary, we must be able to define in the first instance, in order to be capable to reproduce with the resources we have (energy, materials) to reproduce with the fidelity that the current technical means (printers) allow us.

Regarding the definition of the objects to be reproduced, the current $\mathrm{CAD}$ (computer-aided design), which as a result of a set of mathematical calculations related to position and shape, allow endowing the objects that they represent through a graphical interface with a set of attributes some of them superficial and relative to the position, and others more general within the volume they describe. In this aspect, based on the historical roots of subtractive techniques or more traditional conformation, until now we did not need to 
describe the interior of an object since in principle we were not able to perceive in a simple way the interior of a solid element and we were not able to shape the interior of the same with a certain degree of complexity, and in this regard we refer to internal hollow structures with locally variable densities, or constituted of multiple materials arranged in combinations and graduations at will. So, in this sense, it has to be said that nowadays there is no such thing, or at least it is in development but not available software is capable to define, through local attributes, the interior of the objects that it defines and represents. In this aspect, important efforts are carried out by companies such as Autodesk, HP, Dassault Systems, General Electric, Shapeways, SLM Solutions, Microsoft and Siemens, among others, whose objective is to generate common format capable of being used in all the elements of reproduction of three-dimensional pieces. With the name of 3MF Consortium, this group searches under the principles of the AMF file (Additive Manufacturing File Format) to generate a unified format in which it is linked from the design of an element to its printing without going through intermediate conversions where information can be lost along the way, that is: $\mathrm{CAD}+\mathrm{AMF}=3 \mathrm{MF}$ (3D manufacturing format).

Therefore, although the additive manufacturing as a reproduction system can in principle, have the potential to materialize an object through the deposition of materials and by the combination of them and in different graduations, generate locally properties designed at will within the physical domain that makes up the objects. Until the computer tools that allow us to represent and consequently define the objects that users can come to conceive have not been created, researchers will see the capabilities of these reproduction systems depleted, let us call them in this case of additive manufacturing or $3 \mathrm{D}$ printing or rapid prototyping.

Reproduction on the other hand, unlike the definition, intervenes the resources that we have to materialize what is defined and in this sense, we refer to the quality of the materials and the way in which the current technology allows us to manipulate them (deposition of metals in liquid state by example in combination with other elements intolerable to high temperatures seems impossible). So, there are important limitations imposed by the materials and the energy requirements that the manipulation of these needs, under the assumption of having both, and to the extent, that we are able to conceive and request them in the digital definition.

The concept of fidelity must be also highlighted, and in this sense it takes two forms: one, the fidelity of the definition, which ultimately leads to the manipulation of huge amounts of data and consequently to very heavy files, as a result of the discretization of the element defined vectorially by the CAD programs, let us say by means of cubic voxels endowed with multiple attributes beyond the chromatics. And two, the fidelity of reproduction. In this regard, once the definition, discretization and limitations imposed by the available resources (materials and energy) have been overcome, the algorithms of the reproductive system optimize the way they are used to reproduce what is requested. The available means now come into playing, that is, the capabilities of systems (printers) and in this sense; we refer to speed, precision and repetitiveness. In relation to fidelity, the smaller the volumes of the deposited material, the greater the combination of materials we can introduce in a pixel (finite area) or in a voxel (finite volume) and in different graduations, the reproduction of the defined attributes will be closer. Geometrically, on the other hand, smaller voxels allow the reproduction of details with higher resolution and therefore a higher fidelity of the digitally defined object.

Finally, another of the challenges facing these additive techniques of three-dimensional reproduction has to do with manufacturing times, returning to the hypothetical replicator. Although the atom is the smallest constituent unit of matter that has the 
properties of a chemical element, the union of these forms a molecule, such as fullerenes, the third known stable molecular form of carbon, after graphite and diamond, capable of forming new structures such as carbon nanotubes, or graphene, where the carbon atoms are arranged in a hexagonal structure, or through the combination of multiple elements, other organic molecules or bio-molecules such as DNA. The manipulation of these fundamental elements is nowadays impossible or from a dimensionally greater point of view, the manipulation of molecules poses unprecedented complications. The sustentation for example, while proceeding to the assembly of these molecules in the reproduction of the substances that will conform to the voxel and consequently to the element to be built up, they have to lean on something let us call a substrate, which must in turn be inert as well as the environment that surrounds it. On the other hand, another of the challenges that arise is the dimensional jump, the union between the nanoscale and the micrometre scale or even bigger. Finally, relating to the manufacturing times, how many atoms are required to reproduce a molecule, and how many molecules constitute a protein or simply a particle, and how many of these particles will make up a voxel and ultimately how many of these voxels will be needed to reproduce an object, food, tissue or organ? What is the proportion of voxels per second that must be reached and under those precisions, so that the reproductive element is considered productive? Without a doubt, it challenges of enormous complexity.

\section{Conclusions}

As a corollary, we resort to the following arguments to try to explain the place where we are:

First: To be able to reproduce something it is necessary to first define it (models, software).

Second: Once what it wanted is defined, it is necessary to have the resources to carry it out (energy, materials).

Third: Once defined the what (to the state that the technology of our time allows, software) and the with what (available resources), it is necessary now to address the how (the means), in this aspect we refer to the existing technical scaffolding limited by the state of the current technique.

Thus, in response to the question posed earlier in the title; what are we able to print? An answer could be, nowadays what we are able to define in a digital model and therefore previously perceived through our senses (magnified or not), depending on the resources that the current state of technology is able to provide, and limited by the means that the current state of the technique can offer.

So the current state of the art is far from being able to reproduce all the nuances and graduations that through our senses we are able to perceive. But, if hypothetically we could deposit what is needed, quickly and accurately in a spatial domain it would evidence, those limitations are not only imposed by the existing technique, but by our ability to define what is needed to reproduce what is desired. If finally, in this rational exercise, we would start from the assumption that we are able to deposit what is required where it is needed because the resources we have allow us to do so. It would evidence, the need to have the necessary means, with it we refer to operable materials.

Additive manufacturing stands with a very promising future and with the potential to change the world as it evolves, but these technologies pose many challenges and, like few others, the need for the successful integration of an enormous multitude of disciplines.

\section{Acknowledgements}

The authors would like to acknowledge the program Retos Colaborción of the Spanish Ministry of Economy and Competitiveness for its financial support in the research project Nhibrid32D: RTC-2015-3497-7 and NET3D+: RTC-565780137-80137-45-516 (AEI/FEDER, UE). 


\section{References}

[1] Toffler, A. 1980. The Third Wave. New York: Bantam Books.

[2] Wohlers, T., and Gornet, T. 2014. "Wohlers Report 2017: History of Additive Manufacturing." Wohlers Associate, Belgium, 1-23.

[3] Wohlers, T. 2014. "Wohlers Report 2014: Global Reports." Wohlers Associates, Belgium.

[4] Wohlers, T. 2015. "Wohlers Report 2015: Global Reports." Wohlers Associates, Belgium.

[5] Witowski, J., Pędziwiatr, M., Piotr, M., and Budzyński, A. 2017. "Cost-Effective, Personalized, 3D-Printed Liver Model for Preoperative Planning before Laparoscopic Liver Hemihepatectomy for Colorectal Cancer Metastases." Int. J. Comput Assist. Radiol Surq. 12 (12): 2047-54.

[6] Oyewumi, M. O. 2015. "3D Printing Technology in Pharmaceutical Drug Delivery: Prospects and Challenges." J. Biomol. Res. Ther. 4 (4): e141.

[7] Lopes, A., MacDonald, J. E., and Wicker, R. B. 2012.
"Integrating Stereolithography and Direct Print Technologies for 3D Structural Electronics Fabrication." Rapid Prototyp. J. 18 (2): 129-43.

[8] XU, L., Gutbrod, S. R., Bonifas, A. P., SU, Y., Sulkin, M. S., LU, N., Chung, H., JANG, K., LIU, Z., YING, M., LU, C., Webb, R. C., Kim, J., Laughner, J. I., CHENG, H., LIU, Y., Ameen, A., Jeong, J., Kim, G., HUANG, Y., Efimov, I. R., and Rogers, J. A. 2014. "3D Multifunctional Integumentary Membranes for Spatiotemporal Cardiac Measurements and Stimulation across the Entire Epicardium." Nat. Commun. 5: 1-10.

[9] MacDonald, E., Salas, R., Espalin, D., Perez, M., Aguilera, E., Muse, D., and Wicker, R. B. 2014. "3D Printing for the Rapid Prototyping of Structural Electronics." IEEE Access 2: 234-42.

[10] XU Y., WU, X., GUO, X., KONG, B,. ZHANG, M., and QIAN, X. 2017. "The Boom in 3D-Printed Sensor Technology." Sensors (Basel) 17 (5).

[11] Kaku, M. 2011. Physics of OF the Future. New York: Doubleday. 